East African Medical Journal Vol. 80 No. 1 January 2003

EXPERIENCE WITH LAPAROSCOPIC SURGERY AT THE AGA KHAN HOSPITAL, NAIROBI

R.B. Parkar, MBBS, MMed, Consultant Obstetrician, Gynaecologist \& Laparoscopic Surgeon, P.O. Box 520, Sarit Centre, 00606, Nairobi, Kenya, N.G. Thagana, MBChB, MMed, Consultant Obstetrician \& Gynaecologist, P.O. Box 75163, Nairobi, Kenya, R., Baraza, FRCS, Consultant Surgeon, P.O. Box 20713, Nairobi, Kenya and D. Otieno, MBChB, MMed, Consultant Anaesthetist, Kenyatta National Hospital, P.O. Box 19990, Nairobi, Kenya

Request for reprints to: Dr. R.B. Parkar, P.O. Box 520, Sarit Centre, 00606, Nairobi, Kenya,

\title{
EXPERIENCE WITH LAPAROSCOPIC SURGERY AT THE AGA KHAN HOSPITAL, NAIROBI
}

\author{
R.B. PARKAR, N.G. THAGANA, R. BARAZA and D. OTIENO
}

\begin{abstract}
Objective: To outline the experience of laparoscopic surgery at the Aga Khan Hospital, Nairobi, and to determine the acceptability and outcome of the various procedures undertaken laparoscopically.

Design: A retrospective case analysis.

Subjects: Four hundred and seventy eight cases of laparoscopic surgery were undertaken in Nairobi from May 2000 to May 2002 in the presence of the principal author, of these 408 cases $(\mathbf{8 5 . 3 5 \%})$ were performed at the Aga Khan Hospital in Nairobi. A review of these cases and their outcomes are analysed and presented.

Exclusions: Loss of client records, or follow up, all diagnostic procedures and all procedures performed at the three other private hospitals, namely; Nairobi Hospital, Nairobi Womens' Hospital and the M.P. Shah Hospital, have been excluded in this review Results: Minimal access surgery will in time find its place amongst the institutions in Kenya. In the last two years 408 cases were undertaken at the Aga Khan Hospital, Nairobi, involving a pool of 48 consultants obstetricians and gynaecologists and general surgeons. The case load increased from 7.0 cases per month in 2000 to 22 cases per month in 2001. Sixty four point four six percent of the patients spent one night in hospital, while $1.96 \%$ had conversion to laparotomy intraoperatively. Thirty nine point seven percent of the patients had no previous surgery. The surgical procedures performed included laparoscopic adhesiolysis (34.55\%) and tuboplasty (17.89\%) for primary or secondary infertility, 33 cases $(8.08 \%$ ) for the management of ectopic pregnancies, laparoscopic myomectomy $(15.44 \%)$, ovarian cystectomy $(16.91 \%)$, ovarian drilling (4.65\%), laparoscopic assisted vaginal hysterectomy (15.19\%) and total laparoscopic hysterectomy $(\mathbf{2 . 2 0} \%)$. All cases were reviewed by the consultant pool one week after discharge. The major complications encountered included bladder injury $(0.49 \%)$ and gut injury $(0.73 \%)$.

Conclusions: Minimal access surgery in gynaecology and general surgery is gaining remarkable ground worldwide and has tremendous potential in Kenya. It is evident that in trained hands, the common gynaecological operations and certain general Surgical procedures can be undertaken safely, laparoscopically. Minimal access surgery is acceptable to the patients and significantly favourable outcomes have been established in all the cases undertaken, thus far. Patient compliance has been excellent in this series.
\end{abstract}

\section{INTRODUCTION}

Over the last decade the introduction of a multitude of new operative techniques have transformed the scope of modern surgical practice (1). Scores of entirely new operative procedures have been described, documented and recorded and many classical "open" procedures can now be performed in a less invasive and more precise endoscopic route(1). Mastering these newer techniques of operative intervention, without the need for external exposure offers an exciting challenge for the next generation of surgeons(2).
The major advantages of the minimal access approach are now clear and have been documented in a large number of clinical trails(3). It is evident that the avoidance of a laparotomy incision results in less operative pain and less need of analgesia in the patients. With smaller wounds and less pain, patients recovery is remarkable and their return to normal activities relatively shortened $(3,4)$. This also facilitates a shorter hospital stay, and the time spent away from work is considerably reduced regardless of the complexity of the procedures undertaken(4).

Also noted is a significant reduction in post 
operative adhesion formation following minimal access surgery when compared to the commonly advocated "open" procedures(5). For the surgeon the brilliant and magnified views that modern equipment provides allows the precise definition of anatomy and pathology, and with a newer generation of instruments, constantly being developed and re-developed, facilitate accurate surgery(6). These generic advantages apply equally to any intra abdominal operation which would otherwise require a laparotomy(5).

The general uptake of minimal access surgery in gynaecology is disappointingly low. The proportion of hysterectomies for example performed with any form of laparoscopic assistance in the U.K. is less than $4 \%$ and $70 \%$ of all hysterectomies are still being performed via a laparotomy incision (6). Compared to the nearly universal uptake of laparoscopic cholecystectomy by the general surgeons, who actually started off from a zero experience base, the general surgeons have today rapidly overtaken the gynaecologists in performing major interventional laparoscopic surgery(6).

Although a growing number of procedures are being added onto an endless array of surgery undertaken laparoscopically, changing from the familiar "open" procedures to superior, but more challenging laparoscopic procedures is so often discouraged. Minimal access surgery often takes longer than the standard open procedures particularly in early "learning curve" phase. Increased operative times, colleague unwillingness or inability to embrace change, or lack of initiative to mould practices for the benefit of their patients are given as excuses to justify the argument that the standard operative techniques developed as long ago as during the early years of the last century are still the classic operations best suited for their patients(6).

The procedure of laparoscopic entry followed for all cases was standard using a Verres needle, and an initial insufflation pressure of $25 \mathrm{mmHg}$ and a flow rate of IL per minute, followed by laparoscopic confirmation, using a $10 \mathrm{~mm}, 0$ or 30 degree laparoscope through a $10 \mathrm{~mm}$ Flap valve Trochar, to indicate adequate intraperitoneal placement. Secondary punctures $(5 \mathrm{~mm})$ were introduced strictly, under direct vision. The pressures were then maintained at $15 \mathrm{mmHg}$ and the flow rate increased to 20 Litres/minute.

Intraoperative haemostasis was maintained by bipolar desiccation, intracorporhoeal and extra corporhoeal sutures and endoloops. Monopolar cutting or coagulation currents were occasionally used. Irrigation was undertaken using warm normal saline in an aquapurator, while suction was obtained through a standard vacuum twin cannister pump, or the main vacuum line.

Of all the ectopic pregnancies that presented with a significant haemoperitoneum, suction of the clots was first undertaken. In the presence of adhesions, adhesiolysis was done using sharp dissection and or monopolar cautery. Total salpingectomy was then undertaken by either bipolar cauterisation and dissection or by applying three endoloop sutures. In the cases of the two unruptured ectopic pregnancies, a linear salpingotomy was done using a monopolar hook. After the ectopic was evacuated, intracorrporheal sutures were used to appose the serosal surfaces.

Laparoscopic adhesiolysis was undertaken using monopolar and or bipolar coagulation with sharp dissection. In the cases of tuboplasty, and cuff salpingostomy in particular, intracorrporheal sutures were placed so as to maintain the tubal patency.

All the myomectomies were handled by first injecting a vasoconstrictor (POR 8-ORNIPRESSIN) into the capsule, followed by opening up the capsule, followed by opening up the capsule using a monopolar hook electrode, and gentle dissection of the fibroid away from its bed. Haemostasis was maintained by bipolar coagulation, and the defect sutured using interupted intracorrporheal sutures in at least two layers.

Laparoscopic ovarian drilling was done using a monopolar needle electrode. In cases of ovarian cystectomy, the open cystectomy, rather than an intact (or closed) cystectomy was the preferred choice, in which case the cyst was opened, the lining stripped from the ovary and then the ovary sutured, to appose the edges.

Laparoscopic assisted vaginal hysterectomy (LAVH) is becoming the popular option to the total abdominal hysterectomy. In the series presented, the round ligaments, ovarian ligaments and broad ligaments were cauterized with bipolar coagulation and cut using scissors.

The cost of providing adequate funding for suitable equipment for both surgery and training (or rather "retraining") doctors' are further obstacles to the widespread use of minimal access surgery. Although the benefits of laparoscopic surgery can be brought to many patients, and minimal access surgery can be used with advantage for every intra-abdominal operative procedure, such can only be achieved with a sustained interest, adequate training and experience and the availability of modern techniques and equipment(6).

In Kenya, the full potential, and development of minimal access surgery is yet to be realised. In this paper a group of surgeons presents its experience with minimal access surgery over a two year period.

\section{MATERIALS AND METHODS}

A retrospective review of the cases undertaken in the presence of the principle author was conducted. The procedures were performed between May 2000 and May 2002, at various hospitals in Nairobi, (Table 1).

All cases whose records were incomplete or not retrieved, cases in which no operative intervention was undertaken (Diagnostic Procedures) and cases who were lost to follow up were excluded. Cases undertaken at the Nairobi Hospital, Nairobi Womens Hospital and 
the M.P. Shah Hospital were not entered in the study.

Table 1

Laparoscopic Surgery Cases: By Institutions

\begin{tabular}{|c|c|c|c|c|c|}
\hline Period(Years) & $\begin{array}{c}\text { The Aga } \\
\text { Khan } \\
\text { Hospital }\end{array}$ & $\begin{array}{c}\text { The } \\
\text { Nairobi } \\
\text { Hospital }\end{array}$ & $\begin{array}{c}\text { The } \\
\text { Nairobi } \\
\text { Womens } \\
\text { Hospital }\end{array}$ & $\begin{array}{c}\text { M.P. } \\
\text { Shah } \\
\text { Hospital }\end{array}$ & $\begin{array}{l}\text { Totals } \\
\end{array}$ \\
\hline $\begin{array}{l}2000 \\
\text { (May to Dec) } \\
2001 \\
\text { (Jan to Dec) } \\
2002 \\
\text { (Jan to Mav) }\end{array}$ & 260 & 0 & 41 & 0 & 309 \\
\hline (Jan to May) & $\frac{92}{408}$ & 13 & 14 & 0 & 113 \\
\hline
\end{tabular}

At the Aga Khan Hospital, a standard Karl Storz tower consisting of two Sony 21 inch monitors, a Trican SL Pal camera, a Xenon 300 light source, an electronic endoflator, an Autcon 350 high frequency diathermy unit and a Unimat aquapurator were used for all the cases.

\section{Figure 1}

Laparoscopic surgery cases by month $(n=478)$

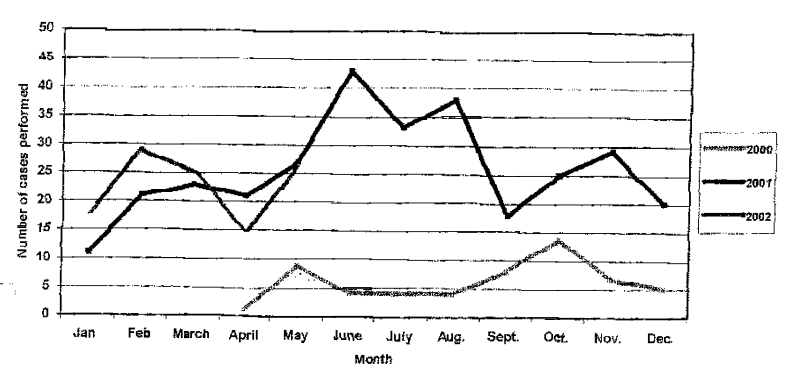

Figure 2

Laparoscopic surgery cases: By Institutions

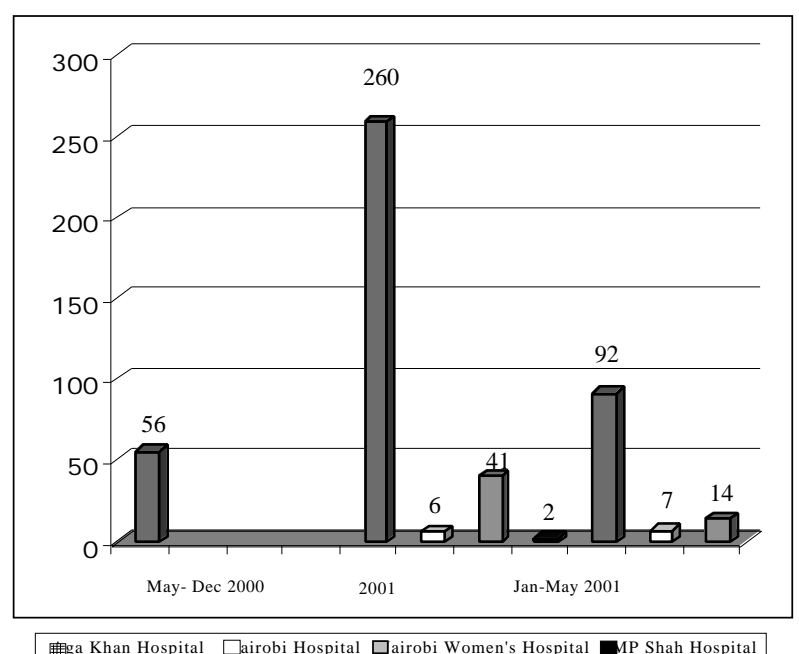

The uterovesicle pouch was opened and the bladder reflected. If the uterine arteries were well skeletonised, then they were sutured laparoscopically. If not then they were tackled vaginally. The rest of the procedure was completed vaginally, after which a re-look with the laparoscope confirmed adequate haemostasis.

Records of the various procedures, operative time, complications and follow up were adequately maintained on a Toshiba Satellite 4070 CDS laptop computer.

The results of $408(85.35 \%)$ of the reviewed cases are presented and discussed herein.

\section{RESULTS}

Four hundred and seventy eight cases were performed from May, 2000 to May 2002 in Nairobi, of these $408(85.35 \%)$ were performed at The Aga Khan Hospital Nairobi, and have been reviewed.

Table 2

Case load per month

\begin{tabular}{|c|c|c|c|c|}
\hline Period(Years) & $\begin{array}{l}\text { Total } \\
\text { Cases }\end{array}$ & $\begin{array}{c}\text { Cases } \\
\text { Reviewed }\end{array}$ & $\begin{array}{l}\text { Average } \\
\text { Cases } \\
\text { per month } \\
\text { (reviewed } \\
\text { cases) }\end{array}$ & $\begin{array}{r}\text { Reviewed } \\
\text { Cases (\%) }\end{array}$ \\
\hline \multirow{4}{*}{$\begin{array}{l}2000 \\
\text { (May to Dec) } \\
2001 \\
\text { (Jan to Dec) } \\
2002 \\
\text { (Jan to May) }\end{array}$} & 56 & 56 & 7.0 & 100 \\
\hline & 309 & 260 & 22.0 & 84.14 \\
\hline & 113 & 92 & 18.00 & 81.41 \\
\hline & 478 & 408 & & \\
\hline
\end{tabular}

The case load increased from seven cases per month in the year 2000 to an average of 22 cases per month in 2001 (Table 2). The maximum cases undertaken in a month were 43 cases in June, 2001. This was apparent as minimal access surgery became more acceptable amongst surgeons, and concomitantly the patient demand increased. In the first five months of the year 2002 (January to May), an average of 18 cases were performed monthly. It is expected that this will increase as we advance towards the end of the year.

Sixty two point seven percent were in the 30 to 45 year age bracket, $38.6 \%$ were para 2 , and $21.2 \%$ were nulliparous, while $3.4 \%$ were para 4 and above.

Three hundred and fifty eight cases $(87.74 \%)$ of the total 408 performed at the Aga Khan Hospital, Nairobi, were referred by a pool of 48 consultants obstetricians and gynaecologists or general surgeons, some of whom have attained adequate experience and are undertaking basic procedures confidently on their own (Table 3). 
Table 3

Consultants pool (Case Referrals)

\begin{tabular}{lccc}
\hline & $\begin{array}{c}2000 \\
\text { (May to } \\
\text { December) }\end{array}$ & $\begin{array}{c}2001 \\
\text { (Jan-Dec) }\end{array}$ & $\begin{array}{c}2002 \\
\text { (May to } \\
\text { December) }\end{array}$ \\
\hline Principle Author & 12 & 53 & 31 \\
Consultant 1 & 35 & 99 & 32 \\
$\quad$ II & 8 & 19 & 6 \\
III & 0 & 24 & 7 \\
Others (45 & $1(78.57 \%)$ & $65(79.61 \%)$ & $16(66.30 \%)$ \\
$\quad$ consultants) & 56 & 260 & 92 \\
\hline Total & & &
\end{tabular}

The operating times for the various surgical procedures ranged from 50 to 310 minutes, the average of 95 minutes attained in $37.80 \%$ of the procedures. It is worthy to note that as more and more cases are undertaken and as the skills of the surgeons are developed, the operating times will gradually reduce and plateau off. Of the 408 cases reviewed, five patients underwent intraoperative conversion to a laparotomy, $(1.22 \%)$ either due to a complication or a difficult procedure. One additional patient had a laparotomy on day seven following intestinal obstruction due to secondary port herniation of the small bowel. In this series $64.46 \%$ (263 patients), spent one night in hospital post operatively, while nine patients $(2.20 \%)$ spent more than four days in hospital.

In $162(39.70 \%)$ of the cases (162), no previous surgery had been undertaken, while in $246(60.30 \%)$ cases some form of previous surgery had been done (Table 4).

Table 4

Relevant previous surgery $(n=408)$

\begin{tabular}{lcc}
\hline & No. & $\%$ \\
\hline No Previous & & \\
Surgery & 162 & 39.70 \\
Diagnostic Laparoscopy & 206 & 50.49 \\
Laparotomy & & 3.4 \\
$\quad$ Ovarian Cystectomy & 14 & 14.46 \\
$\quad$ Tuboplasty & 59 & 3.4 \\
$\quad$ Ectopic Pregnancy & 14 & 11.27 \\
$\quad$ Hysterectomy & 2 & 22.3 \\
Appendicectomy & 46 & \\
Caesarian Sections & 91 &
\end{tabular}

The surgical procedures undertaken in this review included laparoscopic adhesiolysis (34.55\%) and tuboplasty $(17.89 \%)$ for primary or secondary infertility, 33 cases $(8.08 \%)$ for the management of ectopic pregnancies, laparoscopic myomectomy (15.44\%), ovarian cystectomy $(16.91 \%)$, ovarian drilling $(4.65 \%)$, laparoscopic assisted vaginal hysterectomy (15.19\%), and total laparoscopic hysterectomy (2.20\%). In addition 10 laparoscopic cholecystectomies and eight total laparoscopic appendicectomies were undertaken in the presence of their respective general surgeons (Table 5).
Table 5

Operative procedures undertaken laparoscopically at the Aga Khan Hospital $(n=408)$

\begin{tabular}{lc} 
TUBAL & \\
Adhesiolysis & 141 \\
Tuboplasty & 73 \\
Ectopic Pregnancy & \\
$\quad$ Salpingostomy & 10 \\
$\quad$ Salpingectomy & 21 \\
$\quad$ Salpingotomy & 2 \\
Tubal ligation & 19 \\
BTL reversal & 1 \\
OVARIAN & \\
Ovarian biopsy & 2 \\
Ovarian drilling & 19 \\
Cystectomy & 69 \\
Oopherectomy & 4 \\
UTERINE & \\
Myomectomy & 61 \\
Laparoscopic assisted vaginal & \\
hysterectomy & 63 \\
Total & \\
Laparoscopic hysterectomy & 9 \\
OTHERS & \\
Appendicectomy & \\
Cholecystectomy & 10 \\
\hline
\end{tabular}

All cases were reviewed by the respective consultant pool, one week after the operative procedure. There was one case of intraoperative secondary port site bleed, two cases of secondary port haematoma and two cases of primary port (umbilical) abscess (Table 6).

Table 6

Complications of laparoscopic surgery at the Aga Khan Hospital $(n=408)$

\begin{tabular}{lc}
\hline ANAESTHETIC & \\
$\quad$ Postoperative Nausea & $62(15.19 \%)$ \\
$\&$ Vomiting & $89(21.82 \%)$ \\
Muscle Aches & $3(0.73 \%)$ \\
Severe Shoulder Pain & $2(0.49 \%$ \\
Brachial Nerve Palsy & $1(0.24 \%)$ \\
$\quad$ Ventricular Fibrillation & \\
OPERATIVE & \\
Port site & \\
Bleeding & 1 \\
$\quad$ Haematoma & 2 \\
Abscess & 2 \\
$\quad$ Herniation of & 0 \\
small Bowel & \\
$\quad$ Omentum & \\
Gut Injury & \\
$\quad$ Primary & \\
Secondary & \\
Bladder Injury & \\
Laparotomy Conversion & \\
ICU Admission & \\
Mortality & $2(0.73 \%)$ \\
\hline
\end{tabular}




\section{DISCUSSION}

Minimal access surgery is gaining tremendous potential both in gynaecology and general surgery and more specialists are increasingly finding it relevant for various surgical procedures. Yat May Wong (1999) noted that an increasing number of gynaecological procedures are now being competently managed laparoscopically rather than by laparotomy(7). The American Association of Gynaecologic Laparoscopists has recommended that the majority of patients with pelvic pathology requiring surgery can be managed laparoscopically(8). In an earlier publication, Parkar, reviewed the outcome of 596 cases done laparoscopically in Kenya, with an intraoperative conversion to laparotomy of $0.67 \%$ (9). All patients scheduled for laparoscopic surgery should consent for possible intraoperative conversion to laparotomy, since in any given unit 10 to $15 \%$ of all cases may inadvertantly be converted(10).

In this series adhesiolysis of pelvic adhesions, causing compromised fertility accounted for $34.55 \%$ of the procedures due. In 73 patients $(17.89 \%)$, either a cuff or a linear salpingostomy was also performed. Pelvic adhesions secondary to infections are a common pathology in this region, and form a majority in the female with compromised fertility. Lundorff et al(5) documented that postoperative adhesions developed more significantly and more often following a laparotomy than after laparoscopic surgery $(\mathrm{p}>0.0001)$ (5). It has been shown that there is a more favourable outcome when tubal pathology for female infertility is undertaken by laparoscopic surgery rather than by laparotomy(5).

The management of ectopic pregnancies has undergone marked changes in the past decade. Goldner et al (10), reported a four fold increase in the incidence of ectopic pregnancy in the United States between 1970 and 1989 , with a fall in the mortality from 35.5 to 3.8 deaths per 10,000 cases(10). In the United Kingdom the increase of ectopic pregnancies doubled from 4.9 to 9.6 per 1000 pregnancies between 1973 and 1993, whilst the mortality decreased from 16 to 3 per 10,000 cases(11). Sau et al(12) recommended that laparoscopic surgery be the preferred option to laparotomy in the management of haemodynamically stable patients with ectopic pregnancies(12). At the Aga Khan Hospital, Nairobi, 33 cases of ectopic pregnancies $(8.08 \%$ were managed laparoscopically between May 2000 and May 2002. Of these 10 were salpingostomy, 21 salpingectomy, and two salpingotomy. In an earlier review by the principal author a total of $28(4.69 \%)$ ectopic pregnancies were handled laparoscopically out of 596 laparoscopic surgical cases, performed in the coast province of Kenya(9). Yao and Tulandi(13), reported in a randomised controlled trial that there was no doubt that the laparoscopic approach in the management of tubal ectopic gestation is associated with a significantly less blood loss, lower analgesic requirement, shorter hospital stay, lower costs and quicker post operative recovery. The subsequent intrauterine pregnancy rate was $61 \%$ when compared to $52 \%$ after laparotomy. The recurrent ectopic pregnancy rate was also lower in the laparoscopic group (8\%) than following laparotomy (14\%) (13). Sylvia et al. (14) in a prospective study, compared the reproductive outcomes following laparoscopic salpingectomy and salpingostomy. The intrauterine pregnancy rate were $60 \%$ and $53.8 \%$, and the recurrent ectopic rates were $18.3 \%$ and $7.7 \%$ respectively(14). In this series, at the Aga Khan Hospital, 33(8.08\%) cases of ectopic pregnancies were handled laparoscopically out of the 408 cases performed of minimal access surgery. Of these tubal conservation was undertaken in 12 cases while 21 cases had a salpingectomy done. Yap Lip Kee (15) reported a swing of suitable surgery undertaken laparoscopically in the management of ectopic pregnancies from less than 5\% in 1990 to over $93 \%$ in 2000(15). There is no doubt that worldwide today, the laparoscopic management of ectopic pregnancies is justifiably regarded as the gold standard. In our series only two of the 33 cases was ectopic gestation unruptured, the rest had a significant haemoperitoneum evident. The presence of pelvic adhesion can be yet another limiting factor. In view of the above reasons, patients who are haemodynamically compromised, should only undergo laparoscopic intervention if the operating team has acquired adequate experience.

The laparoscopic approach was principally designed to replace the abdominal route. Fernandez et al.(25) noted that before the introduction of laparoscopic assisted vaginal hysterectomy (LAVH) at the Antoine Beclere Hospital in France, $42 \%$ of the hysterectomies were vaginal and $58 \%$ abdominal(25). After the introduction of LAVH, in three years the procedure accounted for $20 \%$ of the hysterectomies which resulted in a concomitant decrease in the rate of abdominal hysterectomy(25). In the series under review 62(15.19\%) cases of laparoscopic assisted vaginal hysterectomy and nine cases $(2.20 \%)$ of total laparoscopic hysterectomies were undertaken. Chaparon and Dubuisson(26) noted that the fact that most hysterectomies for benign disease are still being carried out by the abdominal route is ample justification for laparoscopic surgery to have a role in hysterectomy. Brechin et al. (27) of the Scottish Laparoscopic Surgery Audit Group, evaluated the use of LAVH by a cross section of gynaecologists in Scotland. Of the 505 cases of LAVH done from April 1994 to March 1996, 463 $(91.7 \%)$ were successfully undertaken. Although the patients compliance was remarkable, the intraoperative complications encountered included bladder perforation $(0.6 \%)$, ureteric injury $(0.2 \%)$ and haemorrhage $(2.8 \%)$. The laparotomy conversion was $1.0 \%$. There were no cases of bowel injury(27). 
At the Aga Khan Hospital, Nairobi, there were five $(1.22 \%)$ cases of intraoperative conversion to laparotomy. Post operative nausea and vomiting and muscle aches were encountered in $15.19 \%$ and $21.82 \%$ of the cases respectively. Three $(0.73 \%)$ patients complained of severe shoulder pain postoperatively. Port site complications were encountered in six $(1.47 \%)$ patients. Of concern were the two cases of herniation (one small bowel and one omental). Both patients had laparotomies after day seven postoperatively. Although there were no cases of Class 1 bowel injuries (entry related injuries by verres needle or trochar) in this study, there were $3(0.73 \%)$ cases of Class 11 injuries (injuries acquired whilst operating, during release of dense adhesions) encountered.

Minimal access surgery is gaining tremendous potential in both gynaecology and general surgery. The known rate of intraoperative and postoperative complications in adequately trained hands ranges from $1-5 \%$ and the mortality between 4 to 8 deaths per 100,000 cases(28). Minimal access surgery is generally regarded, as safe, effective, well tolerated by patients, and have numerous advantages over the "open" surgical methods, complications and technical failures must be recognised(29). There is no doubt that complications are directly related to the level of training attained and experience levels so achieved. Querleu et al(30) classified complications in laparoscopic surgery as potentially fatal (vascular, intestinal), or non-lethal intraoperative (urinary tract, minor vessel injury) and postoperative complications (infections, thromboembolism). Bowel injuries have been reported to occur in between $0.1 \%$ to $4 \%$ of cases(30). These injuries are a major cause of morbidity and mortality. Brosens and Gordon (31) in a multinational survey estimated the prevalence of bowel injury to range from 1 in 1652 procedures to 1 in 280 procedures depending on the level of experience attained by the surgeon. The presence of adhesions either from previous surgery infections or endometriosis predisposed to injury. Injury during access or during the surgical procedure were statistically more significant when the surgeon performed fewer than 100 laparoscopies a year(31). In the study under review, the complications rates have been comparative to most of the centres offering high quality minimal access surgery services, worldwide.

Although laparoscopic surgery is now the preferred choice in the successful management of ovarian cysts, there is some concern about the risk of cyst rupture in undiagnosed cases of malignancy. Hulka et al. (8) noted only $53(0.4 \%)$ cases of unsuspected ovarian cancer amongst 13,739 cases of laparoscopic ovarian cyst surgery(8), and the risk is therefore small. In the present series, there were 69 cases $(16.91 \%)$ of ovarian cystectomy undertaken, and with all specimens subjected to histological evaluation there were no cases of malignancy reported. However, there were eight cases of ovarian endometriosis or endometriomas which were confirmed histologically. These cases were adequately handled laparoscopically. There were three cases of dermoid cysts (one twisted and gangerous) which were managed by laparoscopic oopherectomy and delivery by colpotomy. Nineteen cases $(4.65 \%)$ of polycystic ovaries were encountered and adequately handled laparoscopically using a monopolar needle electrode. The feasibility and safety of laparoscopy in the management of ovarian cysts and masses have been well demonstrated and documented $(16,17)$.

Between May 2000 and May 2002, 63 cases of laparoscopic myomectomy were undertaken at the Aga Khan Hospital, Nairobi, the smallest being $2 \mathrm{~cm}$ and largest $18 \mathrm{~cm}$. Three $(4.76 \%)$ were peduculated, 12 $(19.04 \%)$ intramural and the rest $76.20 \%$ were subserous. In general anterior wall or fundal fibroids were easier to remove than those on the posterior wall. The maximum number of fibroids removed were five, although laparoscopic myomectomy was attempted only when there were 2-3 fibroids on the pelvic ultrasound. Myoma extraction from the peritoneal cavity was undertaken by morcellation in 58 cases $(92.06 \%)$, posterior colpotomy in four cases $(6.34 \%)$, and by a minilaparotomy in one case. Haemostasis was adequately achieved in all the cases using bipolar, monopolar coagulation and intracorrporheal sutures. Nehzat et al. in 1991 recommended that myomectomy using the laparoscopic approach was justified provided that patients are carefully selected, the surgeon has had appropriate training, the equipment quality is not compromised and the theatre team is experienced(18). The technique of laparoscopic myomectomy was popularised and refined by Jean Bernard Dubuisson in Paris, who used a combination of monopolar and bipolar electrosurgery and intracorporheal sutures to achieve adequate haemostasis and tissue apposition(19). Miller from Chicago used the ultrasonic (harmonic) scalpel with particular usefulness during myomectomy and made the procedure surprisingly bloodless(20).

Laparoscopic hysterectomies of various types have now been performed for over a decade since they were first reported in 1989 (21). The initial reviews of laparoscopic hysterectomies mentioned complications similar to the standard complications of hysterectomy, including urinary and bladder injuries(22). Worldwide majority of the hysterectomies for benign uterine disease are still being carried out by the abdominal route(22). Dicker et al. (23) and Wilcox et al. (24) in extensive reviews showed that less than $30 \%$ of all hysterectomies were performed vaginally.

The armantareum available to the surgeons of the future increases daily, and no doubt minimal access surgery is the way forward. We as service providers have the responsibility to do what we can so as to develop the safest and most effective forms of treatment for our patients and at the same time be humble to acquire the adequate skills in these newer technologies.

A few disadvantages however do occur in the field 
of minimal access surgery. Majority of the surgeons in Kenya would require additional training and skills development in the modalities of minimal access surgery. As time goes by and more and more specialists are trained in the various methods, minimal access surgery would gain a remarkable reputation in Kenya. It is imperative in any region, where minimal access surgery is still at its infancy, Kenya being an example, that as more and more surgeons develop a sustained interest in these newer modalities of surgery, work collectively as a team and offer the patient load the options of laparoscopic surgery. The acquisition of the basic hardware and operating end instruments into our institutions is yet another limiting factor. However, this will soon be overcome as the awareness increases, client demand persists and more surgeons are stimulated into accepting the viability of minimal access surgery.

Overall as we reach out into the new millenium it would be worthwhile and befitting to see our colleagues taking up these remarkable challenges in our region in time to come, and keeping up with trends worldwide.

\section{ACKNOWLEDGEMENTS}

To all the colleagues who provided the various cases for this study, and the Administration and Staff of The Aga Khan Hospital, Nairobi, The Nairobi Hospital and Nairobi Womens' Hospital and mostly the patients for their contribution, in making this publication a reality.

\section{REFERENCES}

1. Garry, R. Gynaecological Endoscopy. The next 10 years; Editorial, Gynae. Endos. 2002; 11:1-3.

2 Zucker, K.A. Surgical Laparoscopy 4th Edition, Quality Medical Publishing St. Louis, Missouri, 1991.

3. Murphy, A.A., Nager, C.W., Wujek, J.J. et al. Operative laparoscopy versus laparotomy for the management of ectopic pregnancy: A prospective trial. Fertility and Sterility, 1992; 57:1180-1185.

4. Vermesh, M., Silva, P.D., Rosen, G.F., Stein, A.L., Fossum, G.T. and Sauer, M.V. Management of ruptured ectopic pregnancy by linear salpingostomy: a prospective randomised clinical trial if laparoscopy versus laparotomy. Obstet. \& Gynae. 1989; 73:400-404.

5. Lundorff, R., Thorburn, J., Hahlin and Kallfelt, B. Adhesion formation after laparoscopic surgery in tubal pregnancy. A randomised trial versus laparotomy. Fertility and sterility 1998; 55: 911-915.

6. Garry, R. Who is for endoscopic surgery and whom is endoscopic surgery for? Editorial; Gynaec. Endos. 2000; 9:281-283.

7. Wong, Y.M., Saad, A., Tin, C. and Cooke, I.D. Laparoscopic management of ovarian cysts. Gynae. Endos. 2000; 9:7990.

8. Hulka, J., Parker, W., Surrey, M. and Philips, J. Laparoscopic management of ovarian masses AAGL, 1990, Survey. J. Repro. Med. 1992; 7:599-600.

9. Parkar, R.B. Minimal access surgery in gynaecology. Postgraduate Doctor Middle East, 2001; 24:84-92.
10. Goldner, T.E., Lawson, H.W., Xia, Z. and Atrash, H.L.K. Surveillance of ectopic pregnancy: United States, 1970-89, MMWR, CDC Surviellance summaries. 1193; 42:73-85.

11. Department of Health: Report on confidential enquires into maternal deaths in the United Kingdom, 1991-1993, London: HMSO, 1996; 68-73.

12. Sau, A.K., Barr, J.A. and Sau, M. Current Status of management of ectopic pregnancy Gynae. Endos. 1999; 8:73-79.

13. Yao, M. and Tulandi, T. Current status of surgical and non surgical management of ectopic pregnancy. Fertility and Sterility, 1998; 55:911-915.

14 Silva, P.D., Schaper, A.M. and Rooney, B. Reproductive outcome after 143 laparoscopic procedures for Ectopic pregnancy. Obst. \& Gynae. 1993; 81:710-715.

15. Yap Lip Kee. ISGE - Presidents Message. ISGE News, 2002; 9:(Issue 1).

16. Canis, M., Mage, G., Pouly, J.L. et al. Laparoscopic diagnosis of adnexial cystic masses: a 12 year experience with long term follow up. Obstet. Gynae. 1994; 83:707712 .

17. Mettler, L., Irani, S. and Semm, K. Ovarian surgery via pelviscopy. J. Reprod. Med. 1993; 38:130-132.

18. Nezhat, C., Nezhat, F. and Silfens, S. Laparoscopic Myomectomy. Internat. J. Fertility, 1991; 36:275-281.

19. Dubuisson, J.B., Lecuru, F., Foulot, H. et al. Myomectomy by Laparoscopy: A preliminary report of 43 cases. Fertility and Sterility, 1991; 56:827-830.

20. Miller, C.E. Laparoscopic myomectomy in the infertile patient. J. Amer. Assoc. Gynae. Laparos 1996; 4:522-529.

21. Reich, H., Decaprio, J. and Mcglynn, F. Laparoscopic Hysterectomy. J. Gynae. Surg. 1989; 5:213-216.

22. Harris, W.J. and Daniel, J.F. Early Complications of laparoscopic hysterectomy. Obst. Gynae. Survey 1996; 51:559-567.

23. Dicker, R., Greenspan, J. and Strauss, L. Complications of abdominal and vaginal hysterectomy among women of the reproductive age in the United States. Amer. J. Obst. Gynae. 1982; 144:841-848.

24. Wilcox, L., Koowin, L., Pokras, R., Strauss, L., Xia, Z. and Peterson, H. Hysterectomy in the United States, 19881990. Obst. Gynae. 1994; 83:549-555.

25. Fernandez, H., Anquetil, C, Capecla-Allouc, S., Frydman, R. and Gomel, V. Hysterectomy for benign uterine disease: The effect of the introduction of laparoscopic assisted hysterectomy on the selection of Surgical cases. Gynae. Endos. 2000; 9:167-174.

26. Chaparon, C., Dubuisson, J.B. Total Laparoscopic Hysterectomy for benign uterine pathologies with resusable instruments: a safe, reproductive and cost effective procedure. Gynae. Endo. 1996; 5:9-14.

27. Brechin, S., Farquharson, D.M., Hawthorn, R. et al. Scottish laparoscopic surgery audit group. A Scottish audit of laparoscopically assisted vaginal hysterectomy. Gynae. Endos. 2000; 9:233-238.

28. Bongard, F., Dubecz, S. and Klein, S. Complications of therapeutic laparoscopy. Current problems in Surgery, 1994; 31:862-924.

29. Nordestgaard, A.G., Bodily, K.C., Osborne, R.W. and Buttorff, J.D. Major vascular injuries during Laparoscopic procedures. Amer. J. Surg. 1995; 169:543-545.

30. Querleu, D., Chevallier, L., Chapron, C. and Bruhat, M.A. Complications of Gynaecological Laparoscopic Surgery. A French multicentre collaboration study. Gynaec. Endos. 1993; 2:3-6.

31. Brosens, L. and Gordon, K. Bowel Injuries during Gynaecological Laparoscopy: A multinational audit. Gynaec. Endos. 2001; 10:141-145. 